\title{
Approximation of a Laminated Microstructure for a Rotationally Invariant, Double Well Energy Density
}

\author{
Mitchell Luskin * \\ School of Mathematics \\ University of Minnesota \\ Minneapolis, Minnesota 55455 USA \\ Email: luskin@math.umn.edu
}

\begin{abstract}
We give error estimates for the approximation of a laminated microstructure which minimizes the energy $\int_{\Omega} \phi(\nabla v(x)) d x$ for a rotationally invariant, double well energy density $\phi(A)$. We present error estimates for the convergence of the deformation in $L^{2}$, the convergence of directional derivatives of the deformation in the "twin planes," the weak convergence of the deformation gradient, the convergence of the microstructure (or Young measure) of the deformation gradients, and the convergence of nonlinear integrals of the deformation gradient.
\end{abstract}

\section{Introduction}

Martensitic crystals are often found in nature and in the laboratory in a simple laminated state where the crystal structure alternates between two symmetryrelated variants (Burkart and Reid (1953) and Basinski and Christian (1954)). These observations are now well explained by a continuum theory for martensitic crystals based on energy minimization for an energy density which attains a minimum value at several symmetry-related deformation gradients (Ericksen (1986 1987$)$ and Ball and James (1987Г1992)). It is usually also desired that such an energy density be rotationally invariant or frame-indifferent (Ball and James (1987Г1992)) Гso the energy density attains its minimum value on multiple Trotationally invariant energy wells. The simple laminated state found in

${ }^{*}$ This work was supported in part by the NSF through grants DMS 91-11572 and DMS 95-05077, by the ARO through grant DAAL03-92-G-0003, and by a grant from the Minnesota Supercomputer Institute. 
nature is modeled in the continuum theory by energy minimizing sequences of deformations which have layers (with width converging to zero) in which the deformation gradient is nearly constant and across which the deformation gradient oscillates between the energy wells so that the effective energy density becomes the relaxed energy density (seeГfor exampleГKinderlehrer and Pedregal (1991) and Kohn (1991)).

We consider in this paper an energy density with two rotationally invariant energy wells (Kohn (1991) and Ball and James (1992)). The resulting mathematical model gives the laminated microstructure described above. For prescribed boundary conditions which are compatible with this simple laminate $\mathrm{Cit}$ has been proven by Ball and James (1992) that the limiting microstructure (or Young measure) of the deformation gradients for energy minimizing sequences is unique. Although this problem has been widely utilized as a test problem for the numerical approximation of microstructure (see $\Gamma$ for example $\Gamma$ Collins and

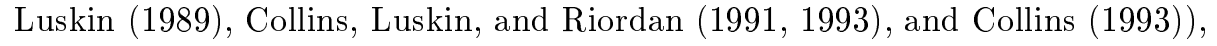
there has not previously been given a proof of convergence or error estimates for any approximation method.

We denote the reference domain for our crystal by $\Omega \Gamma$ which is assumed to be a polygonal domain in $\mathbb{R}^{3}$. We will consider deformations $v: \Omega \rightarrow \mathbb{R}^{3}$ and corresponding deformation gradients $\nabla v(x): \Omega \rightarrow \mathbb{R}^{3 \times 3}$ where $\mathbb{R}^{3 \times 3}$ denotes the space of $3 \times 3$ matrices.

We assume that the energy density $\phi: \mathbb{R}^{3 \times 3} \rightarrow \mathbb{R}$ is a continuous $\Gamma$ nonnegative function. For energy minimizing deformation gradients $F^{+} \in \mathbb{R}^{3 \times 3}$ and $F^{-} \in \mathbb{R}^{3 \times 3}$ एwe define rotationally invariant energy wells by

$$
\mathcal{U}^{+} \equiv \mathrm{SO}(3) \mathcal{F}^{+} \quad \text { and } \quad \mathcal{U}^{-} \equiv \mathrm{SO}(3) \mathcal{F}^{-}
$$

where $\mathrm{SO}(3)$ denotes the space of proper rotations. We then assume that $\phi$ attains its minimum value only on the rotationally invariant set

$$
\mathcal{U} \equiv \mathcal{U}^{+} \cup \mathcal{U}^{-},
$$

so that

$$
\begin{gathered}
\phi(A) \geq 0 \quad \text { for all } A \in \mathbb{R}^{3 \times 3}, \\
\phi(A)=0 \quad \text { if and only if } \quad A \in \mathcal{U} .
\end{gathered}
$$

Since $F^{+}$and $F^{-}$represent symmetry-related states (Ball and James (1987Г 1992)) $\Gamma$ we assume that they satisfy the condition

$$
\operatorname{det} F^{+}=\operatorname{det} F^{-}>0
$$

and the Hadamard condition that there exist $a \in \mathbb{R}^{3}$ and $n \in \mathbb{R}^{3} \Gamma a, n \neq 0$ such that

$$
F^{+}=F^{-}+a \otimes n
$$


where $a \otimes n \in \mathbb{R}^{3 \times 3}$ is the tensor product of $a$ and $n$ defined by $(a \otimes n)_{i j}=a_{i} n_{j}$. We will assume without loss of generality (by rescaling $a$ ) that

$$
|n|=1 \text {. }
$$

We shall see in $\S 3$ that the Hadamard condition (2) allows the existence of a continuous deformation with planar interfaces with normal $n$ (which are called "twin planes" in the physical theory) separating layers in which the deformation gradient is either $F^{+}$or $F^{-}$.

We wish to approximate the microstructure for a simple laminate. Boundary conditions that are compatible for a simple laminate (which is described in more detail in $\S 3$ ) are given by

$$
v(x)=F x \quad \text { for all } x \in \partial \Omega,
$$

where

$$
F=\lambda^{+} F^{+}+\lambda^{-} F^{-}
$$

for $\lambda^{+}, \lambda^{-} \in \mathbb{R}$ satisfying

$$
\lambda^{+}+\lambda^{-}=1 \quad \text { and } \quad 0<\lambda^{ \pm}<1 .
$$

Thus $\Gamma$ we seek to approximate the problem

$$
\inf \left\{\mathcal{E}(v): v \in W^{1, \infty}\left(\Omega ; \mathbb{R}^{3}\right) \cap C^{F}\left(\bar{\Omega} ; \mathbb{R}^{3}\right)\right\}
$$

where the bulk energy of the crystal$\Gamma \mathcal{E}(v) \Gamma$ is defined by

$$
\mathcal{E}(v)=\int_{\Omega} \phi(\nabla v(x)) d x
$$

and where the affine space of continuous functions $\Gamma C^{F}\left(\bar{\Omega} ; \mathbb{R}^{3}\right)$, is defined by

$$
C^{F}\left(\bar{\Omega} ; \mathbb{R}^{3}\right)=\left\{v(x) \in C\left(\bar{\Omega} ; \mathbb{R}^{3}\right): v(x)=F x \text { for } x \in \partial \Omega\right\} .
$$

A theory for the numerical analysis of microstructure was first given by Collins TKinderlerherTand Luskin (1991) and Collins and Luskin (1991) to analyze the minimization of the energy

$$
\hat{\mathcal{E}}(v)=\int_{0}^{1}\left[\phi\left(v^{\prime}(x)\right)+|v(x)-F x|^{2}\right] d x
$$

where $\phi(s): \mathbb{R} \rightarrow \mathbb{R}$ is a double well energy density (see also Luskin (1991)). This theory has been used to analyze the three-dimensional microstructure in micromagnetics (Luskin and Ma (1992)) and extended to some multi-dimensional problems for energy densities which have point energy wells (and so are not rotationally invariant) by Chipot (1991) and by Chipot and Collins (1992). 
A two-dimensional $\Gamma$ rotationally invariant $\Gamma$ double well model with bulk energy

$$
\hat{\mathcal{E}}(v)=\int_{\Omega}\left[\phi(\nabla v(x))+|v(x)-F x|^{q}\right] d x
$$

for $\Omega \subset \mathbb{R}^{2}$ and $1<q<\infty$ has been analyzed by Gremaud (1994) and by Chipot $\Gamma$ Collins $\Gamma$ and Kinderlehrer (1995). The term $|v(x)-F x|^{q}$ in the integrand of (4) is not part of the physical elastic energy density term" which has been added as in the one-dimensional case for mathematical convenience to force the convergence of $v(x) \rightarrow F x$ in $L^{q}\left(\Omega ; \mathbb{R}^{2}\right)$ for energy minimizing sequences of deformations. HoweverTin contrast to the one-dimensional case $\Gamma$ for the multi-dimensional problem the microstructure (or Young measure) for minimizing sequences for the energy (3) is unique even if the elastic energy density does not include the term $|v(x)-F x|^{q}$ (Ball and James (1992)). The results that we prove in this paper do not utilize a term $|v(x)-F x|^{q}$ in the energy density $\Gamma$ but rather show that the convergence of $v(x) \rightarrow F x$ in $L^{2}\left(\Omega ; \mathbb{R}^{3}\right)$ and the convergence of the microstructure depend only on minimizing the elastic energy (3).

The statements of our theorems and lemmas are given in $\S 2$. The remaining sections give the proofs of the theorems and lemmas. An estimate for the minimization of the energy (Theorem 1) is given in $\S 3$. The proofs of the $L^{2}$ convergence of the directional derivatives in the plane orthogonal to $n$ (Theorem 2) and of the $L^{2}$ convergence of the deformation (Lemma 2) are given in $\S 4 \Gamma$ the estimate for the weak convergence of the deformation gradient (Theorem 3) is given in $\S 5$ The estimate for the convergence of the deformation gradient to the set $\left\{F^{+}, F^{-}\right\}$(Theorem 4 ) is given in $\S 6 \Gamma$ the estimate for the convergence of the microstructure (Theorem 5) is given in $\S 7 \Gamma$ and the estimate for the convergence of nonlinear integrals of the deformation gradient (Theorem 6) is given in $\S 8$.

\section{Definitions and Main Results}

We shall assume that $\phi$ grows quadratically away from the energy wells. To describe this precisely $\Gamma$ we let $\pi: \mathbb{R}^{3 \times 3} \rightarrow \mathcal{U}$ be a Borel measurable projection defined by

$$
\|A-\pi(A)\|=\min _{B \in \mathcal{U}}\|A-B\| .
$$

We note that such a projection exists since $\mathcal{U}$ is compact $\Gamma$ although the projection is not uniquely defined at $A \in \mathbb{R}^{3 \times 3}$ where the minimum above is attained at more than one $B \in \mathcal{U}$. We shall assume that there exists $\kappa>0$ such that

$$
\phi(A) \geq \kappa\|A-\pi(A)\|^{2} \quad \text { for all } A \in \mathbb{R}^{3 \times 3}
$$


where we use the matrix norm

$$
\|A\|^{2} \equiv \sum_{i, j=1}^{3} A_{i j}^{2} \quad \text { for } A \in \mathbb{R}^{3 \times 3}
$$

It will also be useful to define the operators $R(A): \mathbb{R}^{3 \times 3} \rightarrow \mathrm{SO}(3)$ and $\Pi: \mathbb{R}^{3 \times 3} \rightarrow\left\{F^{+}, F^{-}\right\}$by the relation

$$
\pi(A)=R(A) \Pi(A) \quad \text { for all } A \in \mathbb{R}^{3 \times 3} .
$$

The operators $R(A)$ and $\Pi(A)$ are easily seen to be uniquely defined by the relation $(6)$.

We shall define the properties required for our finite element spaces abstractly so that they can be applied to the different finite element spaces which have been used to compute microstructure. We assume that $\tau_{h}$ for $0<h<h_{0}$ is a family of decompositions of $\Omega$ into polyhedra $\{K\}$ such that (Quarteroni and Valli (1994)):

1. $\bar{\Omega}=\cup_{K \in \tau_{h}} K$;

2. interior $K_{1} \cap$ interior $K_{2}=\emptyset$ if $K_{1} \neq K_{2}$ for $K_{1}, K_{2} \in \tau_{h}$;

3. if $S=K_{1} \cap K_{2} \neq \emptyset$ for $K_{1} \neq K_{2}, K_{1}, K_{2} \in \tau_{h}$, then $S$ is a common face $\Gamma$ edge $\Gamma$ or vertex of $K_{1}$ and $K_{2}$;

4. $\operatorname{diam} K \leq h$ for all $K \in \tau_{h}$.

We denote our family of finite element spaces by

$$
\mathcal{M}_{h}^{F} \subset C^{F}\left(\bar{\Omega}: \mathbb{R}^{3}\right) \cap W^{1, \infty}\left(\Omega ; \mathbb{R}^{3}\right) \quad \text { for } 0<h<h_{0} .
$$

We assume that there exists an interpolation operator $\mathcal{I}_{h}: C^{F}\left(\bar{\Omega} ; \mathbb{R}^{3}\right) \rightarrow \mathcal{M}_{h}^{F}$ such that

$$
\operatorname{ess}_{\sup } \in \Omega\left\|\nabla \mathcal{I}_{h} v(x)\right\| \leq C \operatorname{ess} \sup _{x \in \Omega}\|\nabla v(x)\|
$$

for all $v \in W^{1, \infty}\left(\Omega ; \mathbb{R}^{3}\right) \cap C^{F}\left(\bar{\Omega} ; \mathbb{R}^{3}\right)$, where the constant $C$ in (7) and below will always denote a generic positive constant which is independent of $h$. We also assume that

$$
\left.\mathcal{I}_{h} v(x)\right|_{K}=\left.v(x)\right|_{K} \text { for } K \in \tau_{h} \text { such that }\left.v(x)\right|_{K} \in\left\{P^{1}(K)\right\}^{3}
$$

where $\left\{P^{1}(K)\right\}^{3} \equiv P^{1}(K) \times P^{1}(K) \times P^{1}(K)$ and $P^{1}(K)$ denotes the space of linear polynomials defined on $K$.

The most widely-used finite element methods based on continuous $\Gamma$ piecewise polynomial spaces have Lagrange interpolation operators $\mathcal{I}_{h}$ which satisfy (7) (for quasi-regular meshes) and (8) (Ciarlet (1978) and Quarteroni and Valli (1994)). In particular $\Gamma(7)$ and (8) are valid for the trilinear elements defined 
on rectangular parellelepipeds which have been extensively used to compute laminated microstructure (Collins and Luskin (1989) $\Gamma$ Collins $\Gamma$ Luskin $\Gamma$ and Riordan $(1991 \Gamma 1993) \Gamma$ and Collins (1993)) $\Gamma$ as well as for linear elements defined on tetrahedra.

For completeness $\Gamma$ we will give in $\S 3$ an extension of the proof by Chipot $\Gamma$ Collins and Kinderlehrer (1995) for the minimization of the energy $\mathcal{E}(v)$ over continuous $\Gamma$ piecewise linear functions to the minimization of the energy $\mathcal{E}(v)$ over the more general family of finite element spaces $\mathcal{M}_{h}^{F}$.

Theorem 1 There exists an optimal approximate deformation, $u_{h} \in \mathcal{M}_{h}^{F}$, such that

$$
\mathcal{E}\left(u_{h}\right)=\min _{v_{h} \in \mathcal{M}_{h}^{F}} \mathcal{E}\left(v_{h}\right) \leq C h^{1 / 2}
$$

The following results will be given for an arbitrary $v_{h}(x) \in \mathcal{M}_{h}^{F}$. This allows the applicability of the results to the local minima with small energy which are typically computed rather than a global minimum (Collins (1993)). We also note that the results hold for arbitrary $v(x) \in W^{1, \infty}\left(\Omega ; \mathbb{R}^{3}\right) \cap C^{F}\left(\bar{\Omega} ; \mathbb{R}^{3}\right)$, so the results are also applicable to more general approximation procedures.

The following lemma shows that the deformation gradients of minimizing sequences converge to the union of the energy wells $\mathcal{U}=\mathcal{U}^{+} \cup \mathcal{U}^{-}$. It follows trivially from (5).

Lemma 1 We have the estimate

$$
\int_{\Omega}\left\|\nabla v_{h}(x)-\pi\left(\nabla v_{h}(x)\right)\right\|^{2} d x \leq \kappa^{-1} \mathcal{E}\left(v_{h}\right) \quad \text { for all } v_{h} \in \mathcal{M}_{h}^{F}
$$

The next theorem demonstrates that the directional derivatives orthogonal to $n$ of sequences of energy minimizing deformations converge strongly.

Theorem 2 For all $v_{h} \in \mathcal{M}_{h}^{F}$ and for all $w \in \mathbb{R}^{3}$ such that

$$
w \cdot n=0 \quad \text { and } \quad|w|=1
$$

we have that

$$
\int_{\Omega}\left|\left(\nabla v_{h}(x)-F\right) w\right|^{2} d x \leq C \mathcal{E}\left(v_{h}\right)^{1 / 2}+C \mathcal{E}\left(v_{h}\right) .
$$

The convergence of the deformation in $L^{2}$ will then follow from the Poincaré inequality and Theorem 2 (see $\S 4)$.

Lemma 2 The deformations $v_{h}(x) \in \mathcal{M}_{h}^{F}$ can be approximated by $F x$ at a rate given by

$$
\int_{\Omega}\left|v_{h}(x)-F x\right|^{2} d x \leq C \mathcal{E}\left(v_{h}\right)^{1 / 2}+C \mathcal{E}\left(v_{h}\right) .
$$


It follows directly from the inequality (10) in Lemma 2 that we can give error estimates for the convergence of $v_{h}(x) \rightarrow F x$ in $L^{2}\left(\Omega ; \mathbb{R}^{3}\right)$ for finite element approximations to the minimization of elastic energies (3) which do not include the term $|v(x)-F x|^{2}$ in the energy density.

The next theorem demonstrates that $\nabla v_{h}(x)$ converges weakly to $F$. We will consider $\omega \subset \Omega$ to be a smooth domain if it has a sufficiently smooth boundary $\partial \omega$ so that the divergence theorem and trace theorem used in its proof are valid.

Theorem 3 For any smooth domain $\omega \subset \Omega$, we have for all $v_{h} \in \mathcal{M}_{h}^{F}$ that

$$
\left\|\int_{\omega}\left(\nabla v_{h}(x)-F\right) d x\right\| \leq C \mathcal{E}\left(v_{h}\right)^{1 / 8}+C \mathcal{E}\left(v_{h}\right)^{1 / 2} .
$$

The proof of Theorem 3 shows that the constants $C$ in Theorem 3 are independent of $h$ and converge to zero as the diameter of $\omega$ converges to zero if $\Gamma$ for example $\Gamma \omega$ is obtained by scaling and translating a reference domain.

The following theorem shows that the deformation gradients of minimizing sequences converge to the set $\left\{F^{+}, F^{-}\right\}$.

Theorem 4 For all $v_{h} \in \mathcal{M}_{h}^{F}$, we have the estimate

$$
\int_{\Omega}\left\|\nabla v_{h}(x)-\Pi\left(\nabla v_{h}(x)\right)\right\|^{2} d x \leq C \mathcal{E}\left(v_{h}\right)^{1 / 2}+C \mathcal{E}\left(v_{h}\right) .
$$

Next $\Gamma$ for any smooth domain $\omega \subset \Omega \Gamma \rho>0 \Gamma$ and $v_{h} \in \mathcal{M}_{h}^{F} \Gamma$ we define the sets

$$
\begin{aligned}
& \omega_{\rho}^{+}=\omega_{\rho}^{+}\left(v_{h}\right)=\left\{x \in \omega: \Pi\left(\nabla v_{h}(x)\right)=F^{+} \text {and }\left\|F^{+}-\nabla v_{h}(x)\right\|<\rho\right\}, \\
& \omega_{\rho}^{-}=\omega_{\rho}^{-}\left(v_{h}\right)=\left\{x \in \omega: \Pi\left(\nabla v_{h}(x)\right)=F^{-} \text {and }\left\|F^{-}-\nabla v_{h}(x)\right\|<\rho\right\} .
\end{aligned}
$$

We will then use Theorem 3 and Theorem 4 to prove the following theorem which describes the convergence of the microstructure (or Young measure) of the deformation gradients of energy minimizing sequences. Theorem 5 states that in any smooth domain $\omega \subset \Omega$ and for any energy minimizing sequence the volume fraction that $\nabla v_{h}(x)$ is near $F^{+}$converges to $\lambda^{+}$and the volume fraction that $\nabla v_{h}(x)$ is near $F^{-}$converges to $\lambda^{-}=1-\lambda^{+}$.

Theorem 5 We have for any smooth domain $\omega \subset \Omega, \rho>0$, and $v_{h} \in \mathcal{M}_{h}^{F}$ that

$$
\begin{aligned}
& \left|\frac{\operatorname{meas}\left(\omega_{\rho}^{+}\left(v_{h}\right)\right)}{\operatorname{meas}(\omega)}-\lambda^{+}\right| \leq C \mathcal{E}\left(v_{h}\right)^{1 / 8}+C \mathcal{E}\left(v_{h}\right)^{1 / 2}, \\
& \left|\frac{\operatorname{meas}\left(\omega_{\rho}^{-}\left(v_{h}\right)\right)}{\operatorname{meas}(\omega)}-\lambda^{-}\right| \leq C \mathcal{E}\left(v_{h}\right)^{1 / 8}+C \mathcal{E}\left(v_{h}\right)^{1 / 2}
\end{aligned}
$$


It can be seen from the proof of Theorem 5 that the constants $C$ in the estimates (11) and (12) are independent of $h$, but depend on $\omega$ and $\rho$.

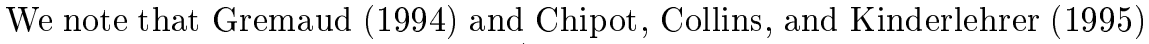
do not give estimates for the sets $\omega_{\rho}^{ \pm}\left(v_{h}\right) \Gamma$ but rather give estimates (for the problem $\int_{\Omega}\left[\phi(\nabla v(x))+|v(x)-F x|^{q}\right] d x$ with $\left.\Omega \subset \mathbb{R}^{2}\right)$ for the sets

$$
\begin{aligned}
& \hat{\omega}_{\rho}^{+}\left(v_{h}\right)=\left\{x \in \omega: \pi\left(\nabla v_{h}(x)\right) \in \mathcal{U}^{+} \text {and }\left\|\pi\left(\nabla v_{h}(x)\right)-\nabla v_{h}(x)\right\|<\rho\right\}, \\
& \hat{\omega}_{\rho}^{-}\left(v_{h}\right)=\left\{x \in \omega: \pi\left(\nabla v_{h}(x)\right) \in \mathcal{U}^{-} \text {and }\left\|\pi\left(\nabla v_{h}(x)\right)-\nabla v_{h}(x)\right\|<\rho\right\} .
\end{aligned}
$$

Thus $\Gamma$ their estimates give the volume fraction that $\nabla v_{h}(x)$ is near the wells $\mathcal{U}^{+}=\mathrm{SO}(3) F^{+}$and $\mathcal{U}^{-}=\mathrm{SO}(3) F^{-}$, whereas Theorem 5 gives an estimate for the approximation of the Young measure since it gives the volume fraction that $\nabla v_{h}(x)$ is near $F^{+}$and $F^{-}$. It is easy to see from Theorem 5 that

$$
\left|\frac{\operatorname{meas}\left(\hat{\omega}_{\rho}^{ \pm}\left(v_{h}\right)\right)}{\operatorname{meas}(\omega)}-\lambda^{ \pm}\right| \leq C \mathcal{E}\left(v_{h}\right)^{1 / 8}+C \mathcal{E}\left(v_{h}\right)^{1 / 2}
$$

since $\lambda^{+}+\lambda^{-}=1$ and since by the definition of $\pi$ we have that $\omega_{\rho}^{ \pm}\left(v_{h}\right) \subset \hat{\omega}_{\rho}^{ \pm}\left(v_{h}\right)$ and $\hat{\omega}_{\rho}^{+}\left(v_{h}\right) \cap \hat{\omega}_{\rho}^{-}\left(v_{h}\right)=\emptyset$.

We next use the analysis introduced in Collins and Luskin (1991) to show that nonlinear integrals of $\nabla v_{h}(x)$ (which approximate macroscopic thermodynamic variables) can be estimated. For linear transformations $\mathcal{L}: \mathbb{R}^{3 \times 3} \rightarrow \mathbb{R}$ we define the operator norm

$$
\|\mathcal{L}\|=\max _{\|A\|=1}|\mathcal{L}(A)|,
$$

so for Lipschitz functions $g(A): \mathbb{R}^{3 \times 3} \rightarrow \mathbb{R}$ we can define the function norm

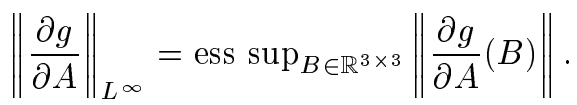

We obtain estimates for the Sobolev space $\mathcal{V}$ of measurable functions $f(x, A)$ : $\Omega \times \mathbb{R}^{3 \times 3} \rightarrow \mathbb{R}$ such that

$$
\int_{\Omega}\left[\left\|\frac{\partial f}{\partial A}(x, \cdot)\right\|_{L^{\infty}}^{2}+|\nabla G(x) \cdot n|^{2}+G(x)^{2}\right] d x<\infty
$$

where

$$
G(x)=f\left(x, F^{+}\right)-f\left(x, F^{-}\right) .
$$

We observe that if $f(x, A) \in \mathcal{V}$, then $f(x, A)$ is Lipschitz continuous as a function of $A \in \mathbb{R}^{3 \times 3}$ for almost all $x \in \Omega$. 
Theorem 6 For all $v_{h} \in \mathcal{M}_{h}^{F}$ and all functions $f(x, A) \in \mathcal{V}$, we have that

$$
\begin{aligned}
& \left|\int_{\Omega} f\left(x, \nabla v_{h}(x)\right)-\left[\lambda^{+} f\left(x, F^{+}\right)+\lambda^{-} f\left(x, F^{-}\right)\right] d x\right| \\
& \quad \leq C\left\{\int_{\Omega}\left[\left\|\frac{\partial f}{\partial A}(x, \cdot)\right\|_{L^{\infty}}^{2}+|\nabla G(x) \cdot n|^{2}+G(x)^{2}\right] d x\right\}^{1 / 2}\left(\mathcal{E}\left(v_{h}\right)^{1 / 4}+\mathcal{E}\left(v_{h}\right)^{1 / 2}\right)
\end{aligned}
$$

where

$$
G(x)=f\left(x, F^{+}\right)-f\left(x, F^{-}\right) .
$$

Finally it follows directly from using the estimate in Theorem 1 that we can obtain the following error estimates for an optimal approximate deformation $u_{h} \in \mathcal{M}_{h}^{F}$ satisfying (9).

Lemma 3 For deformations $u_{h}(x)$ which minimize the energy $\mathcal{E}(v)$ in $\mathcal{M}_{h}^{F}$ and for all $w \in \mathbb{R}^{3}$ such that

$$
w \cdot n=0 \quad \text { and } \quad|w|=1,
$$

we have that

$$
\int_{\Omega}\left|\left(\nabla u_{h}(x)-F\right) w\right|^{2} d x \leq C h^{1 / 4} .
$$

Lemma 4 Any deformation $u_{h}(x) \in \mathcal{M}_{h}^{F}$ which minimizes the energy $\mathcal{E}(v)$ in $\mathcal{M}_{h}^{F}$ is approximated by $F x$ at a rate given by

$$
\int_{\Omega}\left|u_{h}(x)-F x\right|^{2} d x \leq C h^{1 / 4}
$$

Lemma 5 We have for any smooth domain $\omega \subset \Omega$ that if the deformation $u_{h}(x)$ minimizes the energy $\mathcal{E}(v)$ in $\mathcal{M}_{h}^{F}$, then

$$
\left|\frac{\operatorname{meas}\left(\omega_{\rho}^{+}\left(u_{h}\right)\right)}{\operatorname{meas}(\omega)}-\lambda^{+}\right| \leq C h^{\frac{1}{16}}, \quad\left|\frac{\operatorname{meas}\left(\omega_{\rho}^{-}\left(u_{h}\right)\right)}{\operatorname{meas}(\omega)}-\lambda^{-}\right| \leq C h^{\frac{1}{16}} .
$$

Lemma 6 If the deformation $u_{h}(x)$ minimizes the energy $\mathcal{E}(v)$ in $\mathcal{M}_{h}^{F}$ and $f(x, A) \in \mathcal{V}$, then we have that

$$
\begin{aligned}
& \left|\int_{\Omega} f\left(x, \nabla u_{h}(x)\right)-\left[\lambda^{+} f\left(x, F^{+}\right)+\lambda^{-} f\left(x, F^{-}\right)\right] d x\right| \\
& \quad \leq C\left\{\int_{\Omega}\left[\left\|\frac{\partial f}{\partial A}(x, \cdot)\right\|_{L^{\infty}}^{2}+|\nabla G(x) \cdot n|^{2}+G(x)^{2}\right] d x\right\}^{1 / 2} h^{1 / 8}
\end{aligned}
$$

where

$$
G(x)=f\left(x, F^{+}\right)-f\left(x, F^{-}\right) .
$$




\section{Estimates for the Energy}

Proof of Theorem 1. We will now describe a construction of $v_{h}(x) \in \mathcal{M}_{h}^{F}$ such that

$$
\mathcal{E}\left(v_{h}\right)=\int_{\Omega} \phi\left(\nabla v_{h}(x)\right) d x \leq C h^{1 / 2} .
$$

We first define the deformation $w_{h}(x): \Omega \rightarrow \mathbb{R}^{3}$ by

$$
w_{h}(x)=F^{-} x+\left[\int_{0}^{x \cdot n} \chi\left(\frac{s}{h^{1 / 2}}\right) d s\right] a
$$

where $\chi(s): \mathbb{R} \rightarrow \mathbb{R}$ is a characteristic function with period 1 such that

$$
\chi(s)= \begin{cases}1 & \text { for all } 0 \leq s \leq \lambda^{+} \Gamma \\ 0 & \text { for all } \lambda^{+}<s<1\end{cases}
$$

Note that

$$
\left|w_{h}(x)-F x\right| \leq C h^{1 / 2} \quad \text { for all } x \in \Omega .
$$

We also have that

$$
\nabla w_{h}(x)=F^{-}+\chi\left(\frac{x \cdot n}{h^{1 / 2}}\right) a \otimes n, \quad x \in \Omega,
$$

so

$$
\nabla w_{h}(x)= \begin{cases}F^{+} & \text {if } j h^{1 / 2}<x \cdot n<\left(j+\lambda^{+}\right) h^{1 / 2} \text { for some } j \in \mathbb{Z} \\ F^{-} & \text {if }\left(j+\lambda^{+}\right) h^{1 / 2}<x \cdot n<(j+1) h^{1 / 2} \text { for some } j \in \mathbb{Z} .\end{cases}
$$

Next we define the subset

$$
\Omega_{h}^{1}=\left\{x \in \Omega: \operatorname{dist}(x, \partial \Omega)>h^{1 / 2}\right\},
$$

and we define the function $\psi_{h}(x): \Omega \rightarrow \mathbb{R}$ by

$$
\psi_{h}(x)= \begin{cases}1 & \text { for all } x \in \Omega_{h}^{1}, \\ h^{-1 / 2} \operatorname{dist}(x, \partial \Omega) & \text { for all } x \in \Omega \backslash \Omega_{h}^{1} .\end{cases}
$$

The function $\psi_{h}(x)$ is easily seen to satisfy the following properties:

$$
\begin{array}{cl}
0 \leq \psi_{h}(x) \leq 1 & \text { for all } x \in \Omega, \\
\psi_{h}(x)=1 & \text { for all } x \in \Omega_{h}^{1}, \\
\psi_{h}(x)=0 & \text { for all } x \in \partial \Omega, \\
\left|\nabla \psi_{h}(x)\right| \leq C h^{-1 / 2} & \text { for all } x \in \Omega .
\end{array}
$$

We then define the deformation $\hat{v}_{h}(x): \Omega \rightarrow \mathbb{R}^{3}$ by

$$
\hat{v}_{h}(x)=\psi_{h}(x) w_{h}(x)+\left(1-\psi_{h}(x)\right) F x \quad \text { for all } x \in \Omega,
$$


so we have for $x \in \Omega$ that

$$
\nabla \hat{v}_{h}(x)=\left(w_{h}(x)-F x\right) \otimes \nabla \psi_{h}(x)+\psi_{h}(x) \nabla w_{h}(x)+\left(1-\psi_{h}(x)\right) F .
$$

It then follows from $(14) \Gamma(15) \Gamma$ and (16) that

$$
\begin{array}{cl}
\nabla \hat{v}_{h}(x)=\nabla w_{h}(x) \in \mathcal{U}, & x \in \Omega_{h}^{1}, \\
\left\|\nabla \hat{v}_{h}(x)\right\| \leq C, & x \in \Omega, \\
\hat{v}_{h}(x)=F x, & x \in \partial \Omega .
\end{array}
$$

Finally $\Gamma$ we define the deformation $v_{h}(x) \in \mathcal{M}_{h}^{F}$ by

$$
v_{h}(x)=\mathcal{I}_{h}\left(\hat{v}_{h}(x)\right) \text {. }
$$

It follows from the definition of the interpolation operator $\mathcal{I}_{<}$that

$$
v_{h}(x)=\hat{v}_{h}(x)=w_{h}(x) \quad \text { for all } x \in \Omega_{h}
$$

for (recalling that $|n|=1$ )

$$
\Omega_{h}=\Omega_{h}^{2} \backslash \Gamma_{h}
$$

where

$$
\begin{gathered}
\Omega_{h}^{2}=\left\{x \in \Omega: \operatorname{dist}(x, \partial \Omega)>h^{1 / 2}+h\right\}, \\
\Gamma_{h}=\bigcup_{j \in \mathbb{Z}}\left\{x \in \Omega_{h}^{2}:\left|x \cdot n-j h^{1 / 2}\right| \leq h \text { or }\left|x \cdot n-\left(j+\lambda^{+}\right) h^{1 / 2}\right| \leq h\right\} .
\end{gathered}
$$

Now meas $\left(\Omega \backslash \Omega_{h}^{2}\right) \leq C h^{1 / 2}$ since $\Omega \backslash \Omega_{h}^{2}$ is a layer of width $h^{1 / 2}+h$ around the boundary of $\Omega \Gamma$ and meas $\left(\Gamma_{h}\right) \leq C h^{1 / 2}$ since $\Gamma_{h}$ is the union of $\mathcal{O}\left(h^{-1 / 2}\right)$ planar layers of thickness $h$. (Note that only $\mathcal{O}\left(h^{-1 / 2}\right)$ of the sets in the definition of $\Gamma_{h}$ are non-empty.) So $\Gamma$ since $\Omega \backslash \Omega_{h}=\left\{\Omega \backslash \Omega_{h}^{2}\right\} \cup \Gamma_{h}$, we have that

$$
\text { meas }\left(\Omega \backslash \Omega_{h}\right) \leq C h^{1 / 2},
$$

and we have by $(7) \Gamma(15) \Gamma$ and (17) that

$$
\begin{array}{cc}
\nabla v_{h}(x) \in \mathcal{U}, & x \in \Omega_{h}, \\
\left\|\nabla v_{h}(x)\right\| \leq C, & x \in \Omega .
\end{array}
$$

Since $\phi$ is continuous $\Gamma$ it is bounded on bounded sets in $\mathbb{R}^{3 \times 3}$. Thus $\Gamma$ it follows from (18) and (19) that

$$
\begin{gathered}
\left|\int_{\Omega} \phi\left(\nabla v_{h}(x)\right) d x\right|=\left|\int_{\Omega_{h}} \phi\left(\nabla v_{h}(x)\right) d x+\int_{\Omega \backslash \Omega_{h}} \phi\left(\nabla v_{h}(x)\right) d x\right| \\
=\int_{\Omega \backslash \Omega_{h}} \phi\left(\nabla v_{h}(x)\right) d x \leq C h^{1 / 2} .
\end{gathered}
$$




\section{Strong Convergence of the Directional Deriva- tives in the Plane Orthogonal to $n$.}

Proof of Theorem 2. We first observe that

$$
F=\frac{1}{\operatorname{meas}(\Omega)} \int_{\Omega} \nabla v_{h}(x) d x \quad \text { for all } v_{h} \in \mathcal{M}_{h}^{F}
$$

since $v_{h}(x)=F x$ for $x \in \partial \Omega$. Since $F^{+}=F^{-}+a \otimes n$, we have for $w \in \mathbb{R}^{3}$ orthogonal to $n$ that

$$
\begin{aligned}
F^{+} w & =F^{-} w=F w, \\
|\pi(A) w| & =\left|F^{+} w\right|=\left|F^{-} w\right| \quad \text { for all } A \in \mathbb{R}^{3 \times 3} .
\end{aligned}
$$

Hence $\Gamma$ we have for any $w \in \mathbb{R}^{3}$ satisfying $w \cdot n=0$ and $|w|=1$ and any $\mu>0$ that

$$
\begin{aligned}
& \frac{1}{\operatorname{meas}(\Omega)} \int_{\Omega}\left|\left(\nabla v_{h}(x)-F\right) w\right|^{2} d x \\
& \quad=\frac{1}{\operatorname{meas}(\Omega)} \int_{\Omega}\left|\nabla v_{h}(x) w\right|^{2} d x-\frac{1}{\operatorname{meas}(\Omega)} \int_{\Omega}|F w|^{2} d x \\
& \quad=\frac{1}{\operatorname{meas}(\Omega)} \int_{\Omega}\left|\nabla v_{h}(x) w\right|^{2} d x-\left|F^{+} w\right|^{2} \\
& \quad=\frac{1}{\operatorname{meas}(\Omega)} \int_{\Omega}\left|\left[\nabla v_{h}(x)-\pi\left(\nabla v_{h}(x)\right)\right] w+\pi\left(\nabla v_{h}(x)\right) w\right|^{2} d x-\left|F^{+} w\right|^{2} \\
& \leq \frac{1}{\operatorname{meas}(\Omega)} \int_{\Omega}\left(1+\frac{1}{\mu}\right)\left|\left[\nabla v_{h}(x)-\pi\left(\nabla v_{h}(x)\right)\right] w\right|^{2} d x \\
& \quad+\frac{1}{\operatorname{meas}(\Omega)} \int_{\Omega}(1+\mu)\left|\pi\left(\nabla v_{h}(x)\right) w\right|^{2} d x-\left|F^{+} w\right|^{2} \\
& \leq \frac{\mu+1}{\mu \operatorname{meas}(\Omega)} \int_{\Omega}\left\|\nabla v_{h}(x)-\pi\left(\nabla v_{h}(x)\right)\right\|^{2} d x+\mu\left|F^{+} w\right|^{2} \\
& \leq \frac{(\mu+1) \mathcal{E}\left(v_{h}\right)}{\mu \kappa \operatorname{meas}(\Omega)}+\mu\left|F^{+} w\right|^{2} .
\end{aligned}
$$

The result now follows by setting $\mu=\mathcal{E}\left(v_{h}\right)^{1 / 2}$.

We now show that the convergence of the deformation in $L^{2}$ follows directly from the Poincaré inequality (Wloka(1987)) and Theorem 2.

Proof of Lemma 2. Since $v_{h} \in \mathcal{M}_{h}^{F}$, we have that $v_{h}(x)-F x=0$ for $x \in \partial \Omega$. So $\Gamma$ for $w \in \mathbb{R}^{3}$ such that $|w|=1$ we have by integration by parts and the Cauchy-Schwarz inequality that

$$
\begin{aligned}
& \int_{\Omega}\left|v_{h}(x)-F x\right|^{2} d x=-\int_{\Omega}\left(\nabla\left|v_{h}(x)-F x\right|^{2} \cdot w\right)(w \cdot x) d x \\
& \leq 2 \max _{x \in \Omega}|w \cdot x|\left\{\int_{\Omega}\left|v_{h}(x)-F x\right|^{2} d x\right\}^{1 / 2}\left\{\int_{\Omega}\left|\left(\nabla v_{h}(x)-F\right) w\right|^{2} d x\right\}^{1 / 2} .
\end{aligned}
$$


Hence $\Gamma$ we have the Poincaré inequality that for $w \in \mathbb{R}^{3}$ such that $|w|=1$

$$
\int_{\Omega}\left|v_{h}(x)-F x\right|^{2} d x \leq C \int_{\Omega}\left|\left(\nabla v_{h}(x)-F\right) w\right|^{2} d x .
$$

The result of Lemma 2 follows from Theorem 2 and (21) with $w$ choosen so that $w \cdot n=0$ and $|w|=1$.

\section{Weak Convergence of the Deformation Gra- dient}

Proof of Theorem 3. We have by the divergence theorem and the CauchySchwarz inequality that

$$
\begin{aligned}
\left\|\int_{\omega}\left(\nabla v_{h}(x)-F\right) d x\right\| & =\left\|\int_{\partial \omega}\left(v_{h}(x)-F x\right) \otimes \nu d S\right\| \\
& \leq \int_{\partial \omega}\left|v_{h}(x)-F x\right| d S \\
& \leq \operatorname{meas}_{2}(\partial \omega)^{1 / 2}\left(\int_{\partial \omega}\left|v_{h}(x)-F x\right|^{2} d S\right)^{1 / 2}
\end{aligned}
$$

where $\nu$ is the unit exterior normal to $\partial \omega$ and where meas $2(\partial \omega)$ is the surface area of $\partial \omega$. We then use the trace theorem (Adams (1975)) to obtain the estimate

$$
\begin{aligned}
\int_{\partial \omega}\left|v_{h}(x)-F x\right|^{2} d S \\
\leq C \\
\leq C\left[\int_{\omega}\left|v_{h}(x)-F x\right|^{2} d x+\int_{\omega}|\nabla| v_{h}(x)-\left.F x\right|^{2} \mid d x\right] \\
\leq C\left[\int_{\omega}\left|v_{h}(x)-F x\right|^{2} d x+\int_{\omega}\left|v_{h}(x)-F x\right| \cdot\left\|\nabla\left(v_{h}(x)-F x\right)\right\| d x\right] \\
\leq C\left[\int_{\omega}\left|v_{h}(x)-F x\right|^{2} d x\right. \\
\left.+\left(\int_{\omega}\left|v_{h}(x)-F x\right|^{2} d x\right)^{1 / 2}\left(\int_{\omega}\left\|\nabla\left(v_{h}(x)-F x\right)\right\|^{2} d x\right)^{1 / 2}\right] .
\end{aligned}
$$

By the triangle inequality and Lemma $1 \Gamma$ we have that

$$
\begin{aligned}
& \left(\int_{\omega}\left\|\nabla v_{h}(x)-F\right\|^{2} d x\right)^{1 / 2} \\
& \quad \leq\left(\int_{\omega}\left\|\nabla v_{h}(x)-\pi\left(\nabla v_{h}(x)\right)\right\|^{2} d x\right)^{1 / 2}+\left(\int_{\omega}\left\|\pi\left(\nabla v_{h}(x)\right)-F\right\|^{2} d x\right)^{1 / 2} \\
& \quad \leq \kappa^{-1 / 2} \mathcal{E}\left(v_{h}\right)^{1 / 2}+C \operatorname{meas}(\omega)^{1 / 2}
\end{aligned}
$$


since $\|\pi(A)\| \leq C$ for all $A \in \mathbb{R}^{3 \times 3}$ by the compactness of $\mathrm{SO}(3)$.

Hence by using Lemma 2 and (24) in (23) we obtain

$$
\int_{\partial \omega}\left|v_{h}(x)-F x\right|^{2} \leq C \mathcal{E}\left(v_{h}\right)^{1 / 4}+C \mathcal{E}\left(v_{h}\right) \quad \text { for all } v_{h} \in \mathcal{M}_{h}^{F} .
$$

The result of Theorem 3 now follows from using (25) in (22).

\section{Convergence of the Deformation Gradient to $\left\{F^{+}, F^{-}\right\}$}

We have by (20) that for $w \in \mathbb{R}^{3}$ satisfying $w \cdot n=0$

$$
\Pi(A) w=F^{+} w=F^{-} w=F w \quad \text { for all } A \in \mathbb{R}^{3 \times 3} .
$$

Thus Tit follows from (6) and (26) that for $w \cdot n=0$

$$
\begin{aligned}
& {[R(A)-I] F^{+} w=[R(A)-I] \Pi(A) w=[\pi(A)-F] w} \\
& \quad=[\pi(A)-A] w+[A-F] w \quad \text { for all } A \in \mathbb{R}^{3 \times 3} .
\end{aligned}
$$

We then obtain by applying the triangle inequality to (27) with $A=\nabla v_{h}(x)$ and estimating the two terms by Lemma 1 and Theorem 2 that

$$
\begin{aligned}
\int_{\Omega} \mid[ & \left.R\left(\nabla v_{h}(x)\right)-I\right]\left.F^{+} w\right|^{2} d x \\
& \leq 2 \int_{\Omega}\left|\left[\pi\left(\nabla v_{h}(x)\right)-\nabla v_{h}(x)\right] w\right|^{2} d x+2 \int_{\Omega}\left|\left[\nabla v_{h}(x)-F\right] w\right|^{2} d x \\
& \leq C \mathcal{E}\left(v_{h}\right)+C \mathcal{E}\left(v_{h}\right)^{\frac{1}{2}} \quad \text { for } w \cdot n=0 .
\end{aligned}
$$

Next $\Gamma$ since $F^{+}$is nonsingular by (1) $\Gamma$ we can choose $w_{1} \in \mathbb{R}^{3}$ and $w_{2} \in \mathbb{R}^{3}$ to satisfy

$$
\begin{gathered}
w_{1} \cdot n=w_{2} \cdot n=0, \quad F^{+} w_{1} \cdot F^{+} w_{2}=0 \\
\left|F^{+} w_{1}\right|=\left|F^{+} w_{2}\right|=1 .
\end{gathered}
$$

Now $\Gamma$ for

$$
m=F^{+} w_{1} \times F^{+} w_{2},
$$

we have that

$$
Q m=Q F^{+} w_{1} \times Q F^{+} w_{2} \quad \text { for all } Q \in \mathrm{SO}(3) .
$$

SoIfor all $A \in \mathbb{R}^{3 \times 3}$ we have the identity

$$
\begin{aligned}
& {[R(A)-I] m=\left\{R(A) F^{+} w_{1} \times R(A) F^{+} w_{2}\right\}-\left\{F^{+} w_{1} \times F^{+} w_{2}\right\}} \\
& \quad=\left\{[R(A)-I] F^{+} w_{1} \times R(A) F^{+} w_{2}\right\}-\left\{F^{+} w_{1} \times[I-R(A)] F^{+} w_{2}\right\} .
\end{aligned}
$$


Hence $\Gamma$ it follows from the above identity with $A=\nabla v_{h}(x)$ and the estimates (28) that

$$
\int_{\Omega}\left|\left[R\left(\nabla v_{h}(x)\right)-I\right] m\right|^{2} d x \leq C \mathcal{E}\left(v_{h}\right)^{1 / 2}+C \mathcal{E}\left(v_{h}\right) .
$$

Since $\left\{F^{+} w_{1}, F^{+} w_{2}, m\right\}$ is a basis for $\mathbb{R}^{3}$ by (29) and (30) Гwe have from (28) and (31) that

$$
\int_{\Omega}\left\|\left[R\left(\nabla v_{h}(x)\right)-I\right]\right\|^{2} d x \leq C \mathcal{E}\left(v_{h}\right)^{1 / 2}+C \mathcal{E}\left(v_{h}\right) \quad \text { for all } v_{h} \in \mathcal{M}_{h}^{F} .
$$

Theorem 4 is now proven by applying the triangle inequality to the identity

$$
\begin{aligned}
A-\Pi(A) & =[A-\pi(A)]+[\pi(A)-\Pi(A)] \\
& =[A-\pi(A)]+[R(A)-I] \Pi(A) \quad \text { for all } A \in \mathbb{R}^{3 \times 3}
\end{aligned}
$$

with $A=\nabla v_{h}(x)$ and by estimating the two terms by Lemma 1 and (32).

\section{Convergence of the Microstructure}

Proof of Theorem 5. We have that

$$
\begin{gathered}
{\left[\operatorname{meas}\left(\omega_{\rho}^{+}\right)-\lambda^{+} \operatorname{meas}(\omega)\right] F^{+}+\left[\operatorname{meas}\left(\omega_{\rho}^{-}\right)-\lambda^{-} \operatorname{meas}(\omega)\right] F^{-}} \\
\quad=\int_{\omega}\left(\Pi\left(\nabla v_{h}(x)\right)-F\right) d x-\int_{\omega-\left\{\omega_{\rho}^{+} \cup \omega_{\rho}^{-}\right\}} \Pi\left(\nabla v_{h}(x)\right) d x .
\end{gathered}
$$

Now by the triangle inequality $\Gamma$ the Cauchy-Schwarz inequality $\Gamma$ Theorem 3Гand Theorem 4 एwe have that

$$
\begin{aligned}
& \left\|\int_{\omega}\left(\Pi\left(\nabla v_{h}(x)\right)-F\right) d x\right\| \\
& \quad \leq\left\|\int_{\omega}\left(\Pi\left(\nabla v_{h}(x)\right)-\nabla v_{h}(x)\right) d x\right\|+\left\|\int_{\omega}\left(\nabla v_{h}(x)-F\right) d x\right\| \\
& \quad \leq \operatorname{meas}(\omega)^{1 / 2}\left[\int_{\omega}\left\|\Pi\left(\nabla v_{h}(x)\right)-\nabla v_{h}(x)\right\|^{2} d x\right]^{1 / 2}+C \mathcal{E}\left(v_{h}\right)^{1 / 8}+C \mathcal{E}\left(v_{h}\right)^{1 / 2} \\
& \quad \leq C \mathcal{E}\left(v_{h}\right)^{1 / 8}+C \mathcal{E}\left(v_{h}\right)^{1 / 2} \quad \text { for all } v_{h} \in \mathcal{M}_{h}^{F} .
\end{aligned}
$$

Also $\Gamma$ we observe that by the definition of $\omega_{\rho}^{ \pm}\left(v_{h}\right) \Gamma$

$$
\operatorname{meas}\left(\omega-\left\{\omega_{\rho}^{+} \cup \omega_{\rho}^{-}\right\}\right) \leq \frac{1}{\rho} \int_{\omega-\left\{\omega_{\rho}^{+} \cup \omega_{\rho}^{-}\right\}}\left\|\Pi\left(\nabla v_{h}(x)\right)-\nabla v_{h}(x)\right\| d x .
$$


Hence $\Gamma$ since $\|\Pi(A)\|=\left\|F^{+}\right\|$or $\left\|F^{-}\right\|$for all $A \in \mathbb{R}^{3 \times 3} \Gamma$ we can obtain from (34) and Theorem 4 that

$$
\begin{aligned}
& \left\|\int_{\omega-\left\{\omega_{\rho}^{+} \cup \omega_{\rho}^{-}\right\}} \Pi\left(\nabla v_{h}(x)\right) d x\right\| \leq C \operatorname{meas}\left(\omega-\left\{\omega_{\rho}^{+} \cup \omega_{\rho}^{-}\right\}\right) \\
& \quad \leq \frac{C}{\rho} \int_{\omega-\left\{\omega_{\rho}^{+} \cup \omega_{\rho}^{-}\right\}}\left\|\Pi\left(\nabla v_{h}(x)\right)-\nabla v_{h}(x)\right\| d x \\
& \quad \leq \frac{C \operatorname{meas}(\omega)^{1 / 2}}{\rho}\left[\int_{\omega}\left\|\Pi\left(\nabla v_{h}(x)\right)-\nabla v_{h}(x)\right\|^{2} d x\right]^{1 / 2} \\
& \quad \leq C \mathcal{E}\left(v_{h}\right)^{1 / 4}+C \mathcal{E}\left(v_{h}\right)^{1 / 2} .
\end{aligned}
$$

Hence $\Gamma$ we have from (33) and (35) that

$$
\begin{gathered}
\left\|\left[\operatorname{meas}\left(\omega_{\rho}^{+}\right)-\lambda^{+} \operatorname{meas}(\omega)\right] F^{+}+\left[\operatorname{meas}\left(\omega_{\rho}^{-}\right)-\lambda^{-} \operatorname{meas}(\omega)\right] F^{-}\right\| \\
\leq C \mathcal{E}\left(v_{h}\right)^{1 / 8}+C \mathcal{E}\left(v_{h}\right)^{1 / 2} .
\end{gathered}
$$

The result of Theorem 5 now follows from the linear independence of $F^{+}$and $F^{-}$.

\section{Convergence of Nonlinear Integrals of the De- formation Gradient}

Proof of Theorem 6 . We have that

$$
\begin{aligned}
\int_{\Omega}\{f & \left.\left(x, \nabla v_{h}(x)\right)-\left[\lambda^{+} f\left(x, F^{+}\right)+\lambda^{-} f\left(x, F^{-}\right)\right]\right\} d x \\
= & \int_{\Omega}\left[f\left(x, \nabla v_{h}(x)\right)-f\left(x, \Pi\left(\nabla v_{h}(x)\right)\right)\right] d x \\
& \quad+\int_{\Omega}\left\{f\left(x, \Pi\left(\nabla v_{h}(x)\right)\right)-\left[\lambda^{+} f\left(x, F^{+}\right)+\lambda^{-} f\left(x, F^{-}\right)\right]\right\} d x \\
= & \mathcal{J}_{1}+\mathcal{J}_{2} .
\end{aligned}
$$

We estimate the term $\mathcal{J}_{1}$ by Theorem 4 as follows:

$$
\begin{aligned}
\left|\mathcal{J}_{1}\right| & \leq \int_{\Omega}\left\|\frac{\partial f}{\partial A}(x, \cdot)\right\|_{L^{\infty}}\left\|\nabla v_{h}(x)-\Pi\left(\nabla v_{h}(x)\right)\right\| d x \\
& \leq\left[\int_{\Omega}\left\|\frac{\partial f}{\partial A}(x, \cdot)\right\|_{L^{\infty}}^{2} d x\right]^{1 / 2}\left[\int_{\Omega}\left\|\nabla v_{h}(x)-\Pi\left(\nabla v_{h}(x)\right)\right\|^{2} d x\right]^{1 / 2} \\
& \leq C\left[\int_{\Omega}\left\|\frac{\partial f}{\partial A}(x, \cdot)\right\|_{L^{\infty}}^{2} d x\right]^{1 / 2}\left(\mathcal{E}\left(v_{h}\right)^{1 / 4}+\mathcal{E}\left(v_{h}\right)^{1 / 2}\right)
\end{aligned}
$$


Now we have for $G(x)=f\left(x, F^{+}\right)-f\left(x, F^{-}\right)$that

$$
\begin{aligned}
f(x, & \Pi(A))-\left[\lambda^{+} f\left(x, F^{+}\right)+\lambda^{-} f\left(x, F^{-}\right)\right] \\
& =\frac{1}{|a|^{2}}\{a \cdot[\Pi(A)-F] n\}\left[f\left(x, F^{+}\right)-f\left(x, F^{-}\right)\right] \\
& =\frac{1}{|a|^{2}}\{a \cdot[\Pi(A)-F] n\} G(x),
\end{aligned}
$$

so we have from (38) and integration by parts that

$$
\begin{aligned}
\mathcal{J}_{2}= & \int_{\Omega}\left\{f\left(x, \Pi\left(\nabla v_{h}(x)\right)\right)-\left[\lambda^{+} f\left(x, F^{+}\right)+\lambda^{-} f\left(x, F^{-}\right)\right]\right\} d x \\
= & \frac{1}{|a|^{2}} \int_{\Omega}\left\{a \cdot\left[\Pi\left(\nabla v_{h}(x)\right)-F\right] n\right\} G(x) d x \\
= & \frac{1}{|a|^{2}} \int_{\Omega}\left\{a \cdot\left[\Pi\left(\nabla v_{h}(x)\right)-\nabla v_{h}(x)\right] n\right\} G(x) d x \\
& \quad+\frac{1}{|a|^{2}} \int_{\Omega}\left\{a \cdot\left[\nabla v_{h}(x)-F\right] n\right\} G(x) d x \\
= & \frac{1}{|a|^{2}} \int_{\Omega}\left\{a \cdot\left[\Pi\left(\nabla v_{h}(x)\right)-\nabla v_{h}(x)\right] n\right\} G(x) d x \\
& \quad-\frac{1}{|a|^{2}} \int_{\Omega}\left\{a \cdot\left[v_{h}(x)-F x\right]\right\}\{\nabla G(x) \cdot n\} d x .
\end{aligned}
$$

We thus obtain from the Cauchy-Schwarz inequality Lemma $2 \Gamma$ and Theorem 4 that

$$
\left|\mathcal{J}_{2}\right| \leq C\left\{\int_{\Omega}|\nabla G(x) \cdot n|^{2} d x+\int_{\Omega} G(x)^{2} d x\right\}^{1 / 2}\left(\mathcal{E}\left(v_{h}\right)^{1 / 4}+\mathcal{E}\left(v_{h}\right)^{1 / 2}\right) .
$$

The result of Theorem 6 now follows from (37) and (39).

\section{References}

[1] Robert Adams. Sobolev Spaces. Academic Press $\Gamma$ New YorkГ1975.

[2] John Ball and Richard James. Fine phase mixtures as minimizers of energy. Arch. Rational Mech. Anal.Г100:13-52Г1987.

[3] John Ball and Richard James. Proposed experimental tests of a theory of fine microstructure and the two-well problem. Phil. Trans. R. Soc. Lond. АГЗ33::389-450Г1992.

[4] Z. S. Basinski and J. W. Christian. Experiments on the martensitic transformation in single crystals of indium-thallium alloys. Acta Metall. $2: 148$ $166 \Gamma 1954$. 
[5] M. W. Burkart and T. A. Read. Diffusionless phase changes in the indiumthallium system. Trans. AIME J. Metals Г197:1516-1524Г1953.

[6] Michel Chipot. Numerical analysis of oscillations in nonconvex problems. Numer. Math.Г59:747-767Г1991.

[7] Michel Chipot and Charles Collins. Numerical approximations in variational problems with potential wells. SIAM J. Numer. Anal.Г29:1002-1019Г 1992.

[8] Michel Chipot $\Gamma$ Charles CollinsTand David Kinderlehrer. Numerical analysis of oscillations in multiple well problems. Numer. Math.Г 70:259-282Г 1995.

[9] Philippe Ciarlet. The Finite Element Method for Elliptic Problems. NorthHolland $\mathrm{A}$ Amsterdam $\Gamma 1978$.

[10] Charles Collins. Computation of twinning. In J. Ericksen $\Gamma$ R. James $\Gamma$

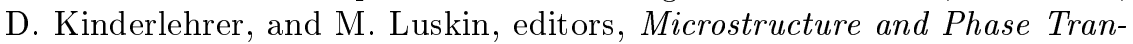
sitions $\Gamma$ pages 39-50ГNew York $\Gamma$ 1993. Springer-Verlag. IMA Volumes in Mathematics and Its Applications $\Gamma$ vol. 54.

[11] Charles Collins DDavid KinderlehrerTand Mitchell Luskin. Numerical approximation of the solution of a variational problem with a double well potential. SIAM J. Numer. Anal.Г28:321-332Г1991.

[12] Charles Collins and Mitchell Luskin. The computation of the austeniticmartensitic phase transition. In Michel RascleГDenis SerreГand Marshall Slemrod $\Gamma$ editors $\Gamma$ Partial Differential Equations and Continuum Models of Phase Transitions $\Gamma$ pages 34-50ГNew YorkГ1989. Springer-Verlag. Lecture Notes in Physics $\Gamma$ vol. 344.

[13] Charles Collins and Mitchell Luskin. Optimal order estimates for the finite element approximation of the solution of a nonconvex variational problem. Math. Comp.Г57:621-637Г1991.

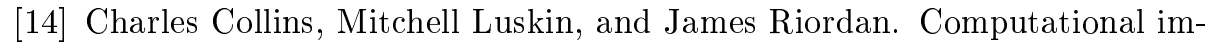
ages of crystalline microstructure. In Jean TaylorГeditorГ Computing Optimal Geometries $\Gamma$ pages 16-18ГProvidence 1991 . Amer. Math. Soc. AMS Special Lectures in Mathematics and AMS Videotape Library.

[15] Charles Collins $\Gamma$ Mitchell LuskinTand James Riordan. Computational results for a two-dimensional model of crystalline microstructure. In J. Er-

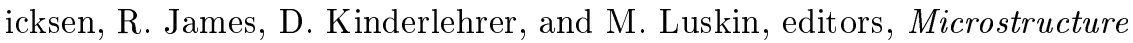
and Phase Transitions $\Gamma$ pages 51-56ГNew York 1993. Springer-Verlag. IMA Volumes in Mathematics and Its Applications $\Gamma$ vol. 54. 
[16] Jerrold Ericksen. Constitutive theory for some constrained elastic crystals. J. Solids and StructuresГ22:951-964Г1986.

[17] Jerrold Ericksen. Some constrained elastic crystals. In John BallГeditorГ Material Instabilities in Continuum Mechanics and Related Problems $\Gamma$ pages 119-137ГOxfordГ1987. Oxford Univ. Press.

[18] Pierre Gremaud. Numerical analysis of a nonconvex variational problem related to solid-solid phase transitions. SIAM J. Numer. Anal.Г31:111-127Г 1994.

[19] David Kinderlehrer and Pablo Pedregal. Characterizations of gradient young measures. Arch. Rat. Mech. Anal.Г115:329-365Г1991.

[20] Robert Kohn. Relaxation of a double-well energy. Continuum Mechanics and Thermodynamics $53: 193-236 \Gamma 1991$.

[21] Mitchell Luskin. Numerical analysis of microstructure for crystals with a nonconvex energy density. In M. Chipot and J. Saint Jean PaulinГeditors $\Gamma$ Progress in Partial differential equations: the Metz Surveys Tpages 156-165Г United Kingdom 11991. Longman House.

[22] Mitchell Luskin and Ling Ma. Analysis of the finite element approximation of microstructure in micromagnetics. SIAM J. Numer. Anal.Г29:320-331Г 1992.

[23] Alfio Quarteroni and Alberto Valli. Numerical Approximation of Partial Differential Equations. Springer-VerlagГBerlinГ1994.

[24] J. Wloka. Partial Differential Equations. Cambridge University Press $\Gamma$ Cambridge $\Gamma 1987$. 